http://jmscr.igmpublication.org/home/ ISSN (e)-2347-176x ISSN (p) 2455-0450

crossref DOI: https://dx.doi.org/10.18535/jmscr/v7i9.89

Journal Of Medical Science And Clinical Research

\title{
Colour Doppler Evaluation of Central Retinal and Ophthalmic arteries in Diabetes patients and Comparing it with Normal Individuals
}

\author{
Authors \\ Sumeena Shanmugam ${ }^{1}$, Ushanandhini.G ${ }^{2}$, Devimeenal.. ${ }^{3}$ \\ ${ }^{1,2}$ Asst Professor, Department of Radiodiagnosis \\ ${ }^{3}$ Professor, Department of Radiodiagnosis \\ Government Kilpauk Medical College, Chennai, Tamil Nadu, India
}

\begin{abstract}
Aim: Aim of the study is to evaluate the ocular blood flow haemodynamics in patients with diabetes mellitus with no ocular symptoms and comparison of the same with normal controls by Doppler imaging.

Materials and Methods: Colour Doppler evaluation of 73 diabetic patients (146 eyes) and 73 healthy controls (146 eyes) were done with Voluson GE machine with High frequency probe (7.5 Mega Hertz). Doppler spectral analysis of ophthalmic arteries $(O A)$ and central retinal arteries $(C R A)$ were done. The peak systolic velocity $(P S V)$, end diastolic velocity $(E D V)$, resistive index $(R I)$ and $S / D$ ratio were calculated. PSV, EDV, RI were measured in normal controls and in diabetics in both the eyes and they were compared. Differences within the groups were evaluated by paired-t-test. A value of less than 0.05 were considered as statistically significant.

Results: The average mean RI of CRA in controls on right side is $0.71+/-0.02$ and 0.69+1-0.02.on left side. The average mean RI of OA in controls on right side is 0.77+1-0.15 and 0.76+1-0.02. on left side. In diabetics the average mean RI of CRA on right side is $0.77+1-0.03$. and $0.76+1-0.11$ on left side. The average mean $R I$ of $O A$ in diabetics on right side is $0.79+1-0.02$ and $0.79+1-0.23$ in left side. The PSV of $C R A$ in diabetics was significantly reduced $(p=<0.05)$ when compared to controls. The EDV of CRA in diabetics was also significantly reduced $(p=<0.05)$ compared to normal controls. The RI is significantly increased compared to normal controls $(p=<0.05)$. 95\% confidence interval is observed in PSV, EDV, RI of CRA in diabetics. The PSV, EDV and RI of OA has no significant difference between normal controls and diabetics.

Conclusion: There were statistical significant difference between the PSV, EDV and RI of CRA in normal and in diabetics. This significant difference could be due to the circulatory changes in blood vessels in diabetics. No significant difference was made in OA between normal controls and in diabetics. This study concludes that retinal haemodynamic changes were present even before the clinical manifestations of retinopathy in diabetics
\end{abstract}

Keywords: Colour Doppler, diabetes, central retinal artery, ophthalmic artery, haemodynamics, resistance.

\section{Introduction}

Diabetes is a disease in which the pancreas is not producing the insulin properly or the body is not utilizing the insulin properly. This leads to increased concentration of glucose in the blood (hyperglycemia). Throughout the world as of 
2010, around 285 million people had diabetes and $90 \%$ of the cases were type 2 . The incidence of diabetes is increasing and it is estimated to almost double by 2030. The prevalence is expected to occur more in Asia and Africa.

\section{Types}

Type 1: Also known as insulin dependent diabetes mellitus. Loss of insulin producing beta cells in pancreas. Children and adolescent groups are most often affected. It is less common type and requires lifelong insulin injections.

Type 2: Also known as Non- Insulin-dependent Diabetes mellitus (NIDDM). It is due to insulin resistance or combined with decreased insulin secretion. Usually occurs in adult hood and can be related to obesity, lack of physical activity and unhealthy diet. It is more common type.

Gestational diabetes: Hyperglycemia occurring during pregnancy.

\section{Complications of diabetes mellitus}

Complications occur in all forms of diabetes mellitus who have no control of blood sugar levels or who are diabetics for longer duration ie $10-20$ yrs. Complications can be microvascular or macrovascular. Macrovascular complications includes cardiovascular disease such as heart attacks, strokes and insufficiency of blood flow to legs. Microvascular complications are due to damage to small blood vessels. It can affect the eyes, kidneys and nerves.

\section{Ocular complications in diabetes}

The primary part affected in eye is retina. Retinopathy is the leading cause of blindness in adults.

Anatomy Of Retina Retina is a sensory tissue which lines the back of the eye. It is multilayered (10 layers) and contains photoreceptors namely rods and cones. The rods and cones convert light energy into signals which are then carried to brain through optic nerves and interprets the signal as visual images. Tiny blood vessels in retina take the oxygen and essential nutrients to the walls of the retina. In the centre of the retina, there is a simple dimple called fovea, which is responsible for the sharp vision in eye. Optic nerve is a collection of nerve fibres which carries electric signals from retina to brain. Retina is supplied by central retinal artery which supplies the inner retinal layer and choroidal arteries which supplies the outer retinal layers. Central retinal artery is a branch of ophthalmic artery. Choroidal artery is a branch of posterior ciliary arteries.

\section{Retinal Microvascular Dysfunction In Diabetes Mellitus}

Microvascular changes occurs early in diabetes mellitus, before the development of disease in succeptible end organs like kidneys and eyes. Microvascular leakage occurs and occlusion of inner-blood retinal barrier occurs. Alterations of haemodynamic parameters in diabetic patients has clinical significance in early diagnosis and treatment of diabetes mellitus microangiopathy. The tissue and nerve cells nourished by small blood vessels are damaged by increasing blood sugar levels.

\section{Retinal complications of diabetes mellitus}

Long standing diabetes can damage the retina leading to blindness. Damage to retina may begin to develop even before the diagnosis of diabetes mellitus. Apart from retinopathy, diabetes mellitus can cause cataract and glaucoma at younger age.

\section{Types of retinopathy}

According to national eye institute, diabetic retinopathy has 4 stages. Mild non prliferative retinopathy-It is an early stage in which tiny blood vessels in the retina swells like a balloon. Moderate non - proliferative retinopathy some blood vessels which supply the retina are blocked. Severe - non - proliferative retinopathy - The retinal blood vessels which are blocked were disrupted and signals the body to produce new blood vessels. Proliferative retinopathy - New blood vessels form either at disc or elsewhere.

In stage 1,2 and sometimes even in stage 3, patients may not have clinical symptoms, but microvascular haemodynamic alterations have occurred in retina even before the symptoms develops. Microvascular changes can be identified 
by standard procedures like fundus examination. But in case of cataract or vitreous haemorrhage fundus examination may not be possible. In a study it was found that longer the duration of diabetes, more is the risk for developing retinal complications. After 5 years $-25 \%$ of IDDM has some retinopathy. After 10 years $-60 \%$ have retinopathy. After 15 years $-80 \%$ have retinopathy.

\section{Retinal artery ultrasound}

Colour Doppler imaging was first used to image various organ systems in 1979. Later in 1989, colour Doppler imaging in orbit was described by Erick son. Eye is located superficially and cystic in nature. The normal anatomy and vasculature can be clearly seen by high frequency transducers. Colour Doppler imaging of eye is a non-invasive procedure. It allows to visualize the grey scale imaging and colour coded imaging both at the same time. ${ }^{([1[-[3])}$ The (peak systolic and end diastolic velocities of the ophthalmic and central retinal arteries can be measured using Doppler. The resistive index can then be calculated using peak systolic and end diastolic velocities. Orbital blood flow velocity can be qualitatively and quantitatively measured by colour Doppler imaging. ${ }^{[4]-[7])}$ Orbital blood flow in normal and in diseased condition can be evaluated. As Doppler shift detection sensitivity is higher than conventional grey scale resolution, evaluation of very small vessels supplying the orbit can be done non invasively. Wolfgang E. Lieb et al examined 40 normal eyes and they were able to locate the central retinal artery (CRA), posterior ciliary arteries (PCA) and ophthalmic arteries (OA) in all patients. Using Doppler spectrum, the blood flow velocity in these vessels are assessed quantitatively. ${ }^{([8]-[10])}$

\section{Materials and Methods \\ Inclusion Criteria}

This study consists of 73 diabetic patients (146 eyes) and 73 healthy controls.(146 eyes). 73 diabetic patients, with deranged glucose tolerance test were the cases for the study. 73 normal persons with normal glucose tolerance without ocular complaints were considered controls. Informed consent were obtained from all the participants and the study protocol was approved by institutional ethics committee.

All diabetic patients were receiving the antidiabetic drugs as usual on the day of scan. Ultrasound examination performed with subjects in supine position. GE voluson machine with 7.5 mega hertz probe was used. Ultrasound probe was positioned gently on closed eyelids in transverse position. Colour Doppler mode was used to identify the central retinal artery (CRA) and ophthalmic artery (OA).

Optic nerve is localized to examine CRA, which runs in centre of the optic nerve along with central retinal vein. Ophthalmic artery OA is situated either above or below the optic nerve in the posterior orbit. Real time identification of CRA and OA was done. Doppler spectral analysis of $\mathrm{OA}$ and CRA was done. Sample volume was estimated by placing the cursor along the course of CRA, 3mm behind the optic disc surface. Sample volume was adjusted to $10 \mathrm{~mm}$ or below. Angle of insonation is adjusted to be $<30$ degree preferably 0 degree. The threshold level was set to optimise sensitivity without producing excessive noise. The peak systolic velocity, end diastolic velocity, resistive index and S/D ratio were calculated. Cross sectional area of CRA and OA were not done because the diameter of these vessels were too small. Peak systolic velocity, End diastolic velocity and Resistive index were measured in normal controls and in diabetics in both the eyes and they were compared. ${ }^{[1]}$

\section{Central Retinal Artery and its Normal Wave} Form Pattern

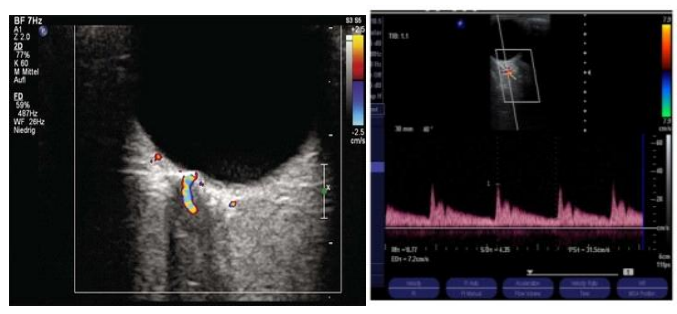


Ophthalmic Artery And Its Normal Wave Form pattern

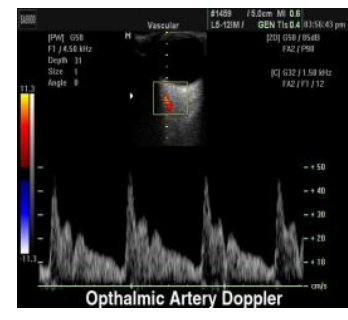

\section{Results-Group 1 Controls}
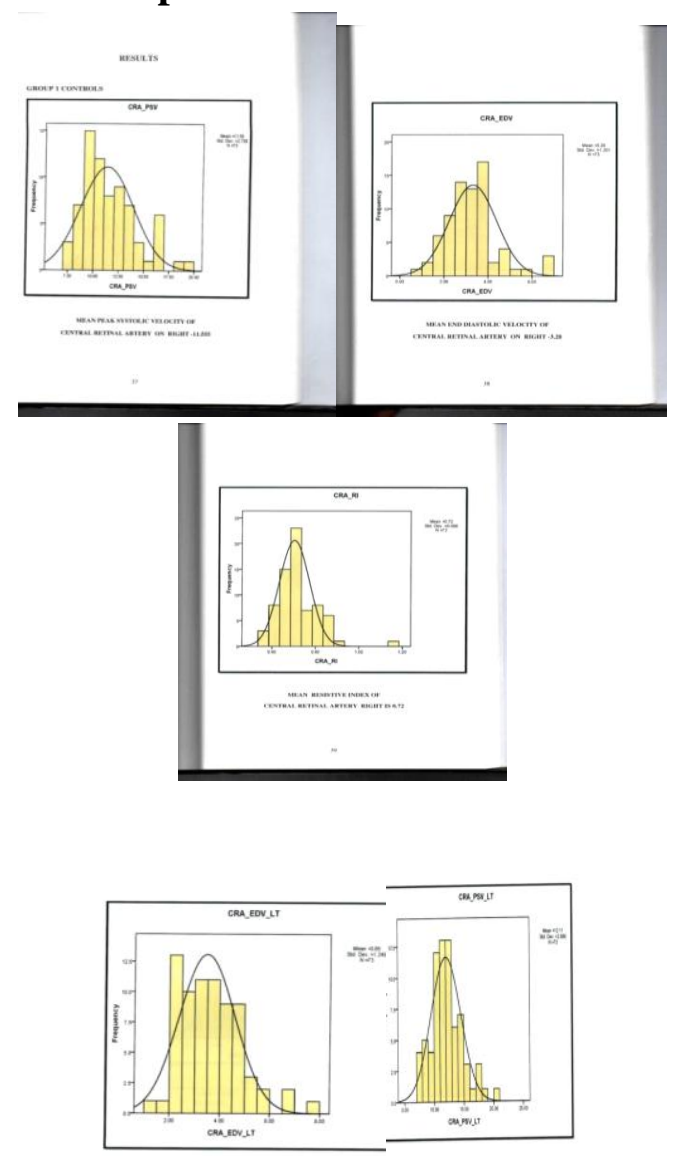

1.
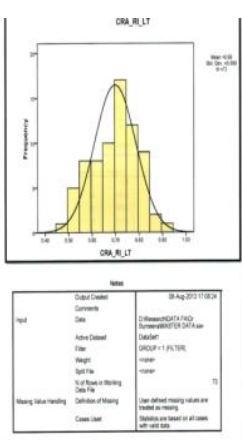

GROUP-2 CASES (DIABETES)
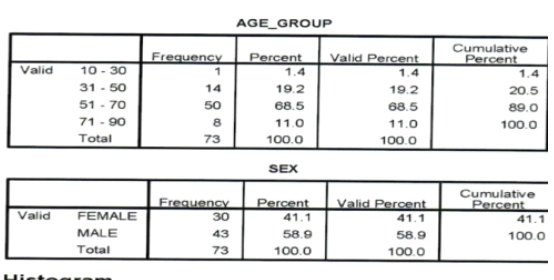

Histogram

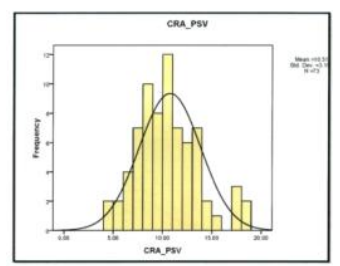

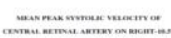

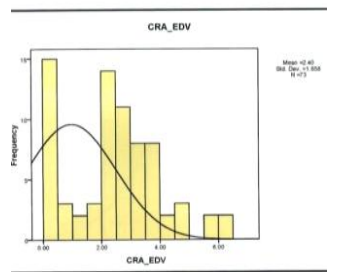

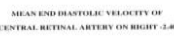
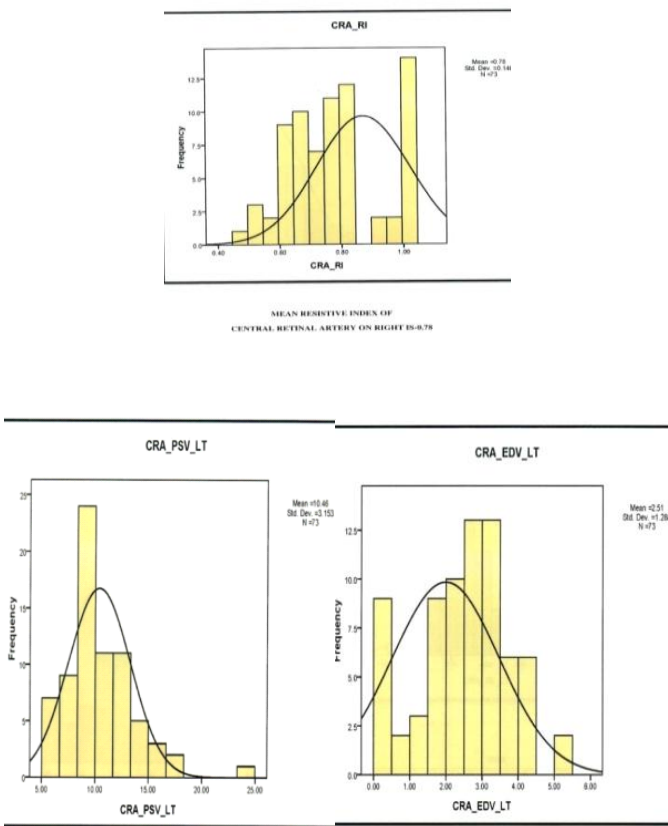

MEAN PEAK SISTOLC vELOCTV OF

MEANEsD Dustouc veoctry of 

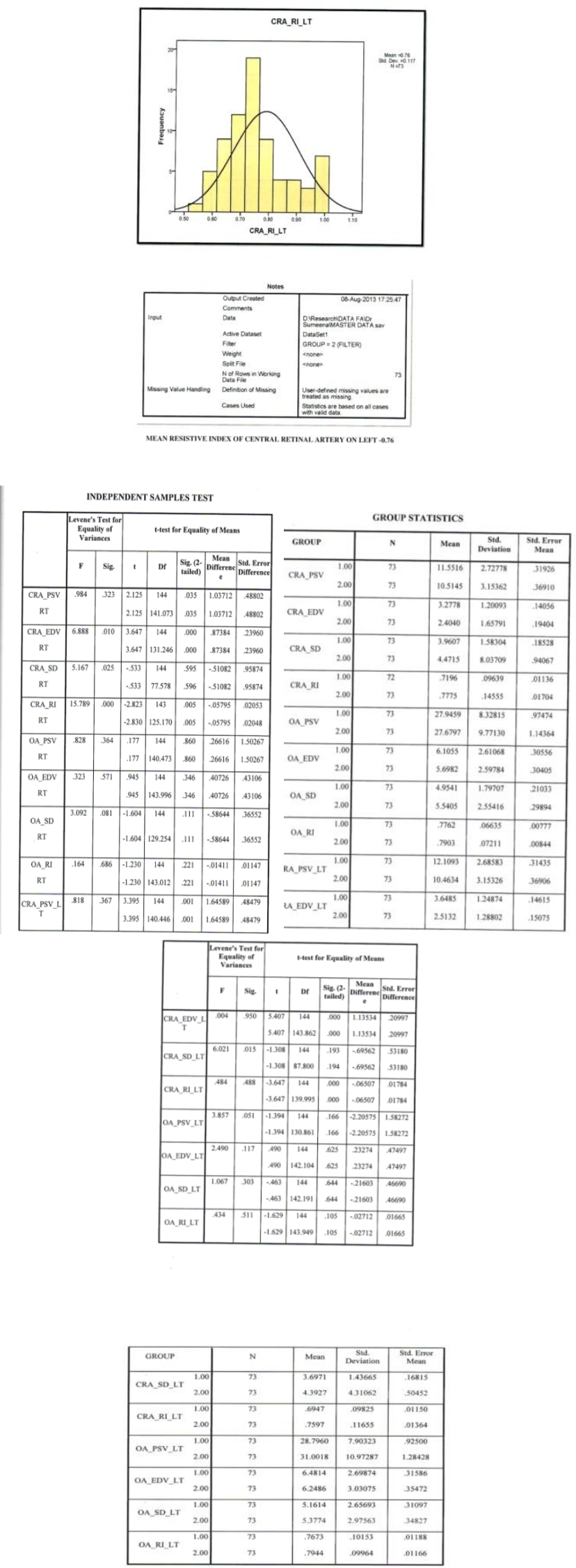

GRoUP1-CONTROL
GROUPC-CASESOLABETES
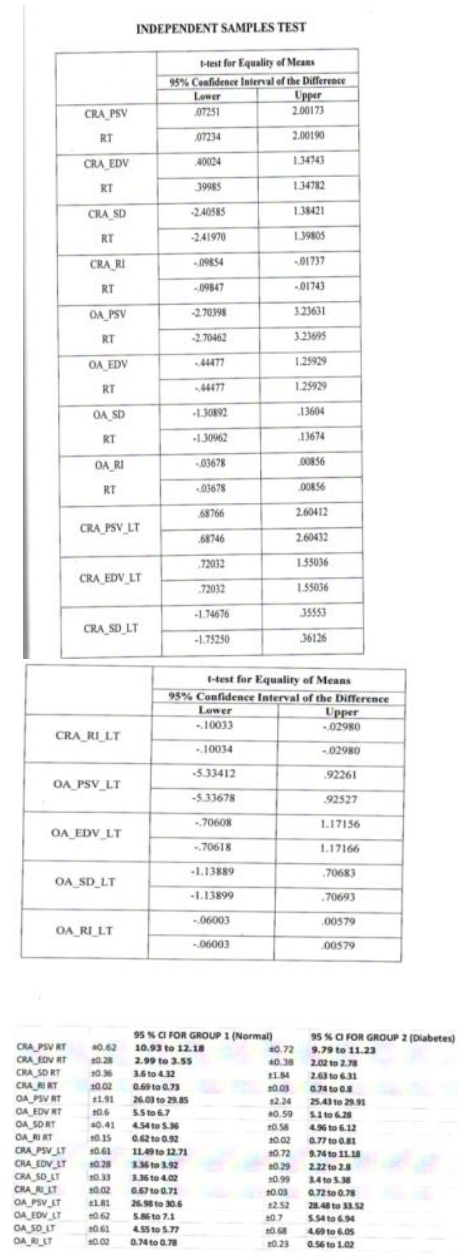

\section{Discussion}

Colour Doppler imaging is a new technique to assess the flow velocity in central retinal arteries and ophthalmic arteries. Doppler ultrasound has been used to evaluate the CRA and OA. It is possible to measure the PSV, EDV and RI of the orbital vessels. ${ }^{\text {[12]-[14]) }}$

The study population consists of 73 normal and 73 diabetic patients. None of them were hypertensives. All the diabetic patients were taking medications on the day of the study. Control subjects were receiving no medications and has no e/o hypertension, diabetes or retinal vascular disease. Ultrasound examinations were performed with subjects in supine position. Using high frequency probe placing over the closed eyelids, central retinal artery and ophthalmic arteries were identified using colour Doppler. PSV, EDV and RI were obtained. 
RI= PSV-EDV/PSV. RI=Resistive index, PSV= Peak systolic velocity, EDV=End diastolic velocity.

RI has been used as a measure of vascular resistance in the artery.

Datas are expressed as the mean +1- SD. Differences within the groups were evaluated by paired-t- test. A value of less than 0.05 were considered as statistically significant. Among the controls 34 subjects were females and 39 of them were males. $9.59 \%$ of them were below $30 \mathrm{yrs}$. $28.77 \%$ of them were $31-50$ yrs.51.37\% of them were $51-70 y r s .10 .27 \%$ of them were $71-90 \mathrm{yrs}$. Among the diabetics 43 were male and 30 were female. $19.2 \% \quad(n=14)$ of them were $31-50$ yrs.68.5\% $(\mathrm{n}=50)$ of them were $51-70$ yrs. $11 \%$ $(n=8)$ of them were 71-90yrs.

17 patients were diabetics for more than $10 \mathrm{yrs}$. Among 73 diabetics, 72 of them were type- 2 and only one patient is type-1. In controls the average mean PSV of CRA on right is 11.55+/-0.62, EDV is $3.27+/-0.28$ and RI is $0.71+/-0.02$. The average mean PSV of CRA on left is $12.10+/-0.61$ and EDV is $3.64+/-0.28$, RI is $0.69+/-0.02$. The average mean PSV of OA on righ is $27.94+1-1.91$ EDV is $6.10+/-0.6$ and RI is $0.77+1-0.15$. The average mean PSV of OA on left is $28.79+/-1.81$ and EDV is $6.48+/-0.62$, RI is $0.76+/-0.02$.

In diabetics, the average mean PSV of CRA on right is $10.51+/-0.72$, EDV is $2.40+/-0.38$ and RI is $0.77+/-0.03$. The average mean PSV of CRA on left is $10.4+/-0.72$, EDV is $2.51+/-0.29$ and RI is $0.76+/-0.11$. The average mean PSV of OA on right is $27.67+/-2.24 \mathrm{EDV}$ is $5.69+/-0.59$ and $\mathrm{RI}$ is $0.79+/-0.02$.The average mean PSV of OA on left is $31.00+/-2.52$ and EDV is $6.24+/-0.7$, RI is $0.79+/-0.23$.

The PSV of CRA in diabetics was significantly reduced $(\mathrm{p}=<0.05)$ when compared to controls. The EDV of CRA in diabetics was also significantly reduced $(\mathrm{p}=<0.05)$ compared to normal controls. The RI is significantly increased compared to normal controls $(\mathrm{p}=<0.05) .95 \%$ confidence interval is observed in PSV,EDV,RI of CRA in diabetics.
The PSV, EDV and RI of OA has no significant difference between normal controls and diabetics. In previous studies, by measuring the blood flow velocities in $\mathrm{OA}$ the severity of diabetic retinopathy was assessed. ${ }^{([15]-[17])}$ However, in this study there is no significant difference in PSV of OA between normal controls and in diabetics . Diastolic flow was absent in 14 out of 73 diabetic patients and this could be due to increased resistance in the distal vascular bed.

Thickening of basement membrane, narrowing or obstructive changes in choroidal capillaries, diabetes duration of 2-9years plays a role in increasing the RI. HbAlc of all the diabetic patients were evaluated. Patients with levels of 7.5 and above has increased subendothelial deposition could contribute to the increased resistance in peripheral vascular bed.

Fake and his coworkers reported that blood flow velocity in retinal arteries were low even before the clinical appearance of retinopathy.

So many studies have reported altered blood flow parameters in retrobulbar bed in diabetic patients without retinopathy. Detecting microvascular changes in retina at the earliest stage is necessary, because this could allow early therapeutic interventions and disease monitoring. ${ }^{[18-20]}$ Patients with diabetes of more than 10 years has RI more than that of patients with slightly higher HbA1c value compared with diabetics who has a good control of their sugar levels with $\mathrm{HbAl} \mathrm{c}$ below 6.5.All the diabetic patients were screened with fundus examination to look for retinopathy changes. Out of 73 diabetics only 2 patients had early retinopathy changes, but clinically they had no symptoms. Using colour Doppler it is shown that the peaksystolic velocity and the end diastolic velocity is significantly reduced and the resistive index is signifantly increased in diabetics compared to normal controls.

This stastically significant difference in peaksystolic velocity and the end diastolic velocity and resistive index could be due to haemodynamic alterations that occur in diabetics. This haemodynamic alteration could have 
occurred in diabetic patients even before the clinical manifestations.

\section{Advantages}

Ultrasound can be done effectively in patients who are affected by cataract and intraocular haemorrhage. Sometimes OA and PCA can be inaccessible by flourescein angiography and Doppler velocimetry. During such situations colour Doppler imaging can be done.

\section{Conclusion}

By using, colour Doppler, the flow velocity in CRA and OA was calculated in diabetics and in normal controls and they were compared. There were statistical significant difference between the PSV, EDV and RI of CRA in normal and in diabetics. This significant difference could be due to the circulatory changes in blood vessels in diabetics. No significant difference was made in OA between normal controls and in diabetics. This study concludes that retinal haemodynamic changes were present even before the clinical manifestations of retinopathy in diabetics.

\section{References}

1. Lieb WE. Flaharty PM, HOA, Sergott RC. Acta ophthalmol 1992 (204); 50 -4. Colour Doppler imaging of eye and orbit. A synopsosis of 400 cases experience.

2. Dudea SM, M.ed ultrason 2011 jun 13 (2) 171-4. Ultrasonography of eye and orbit.

3. T subakimori.s, Fukuda M. Colour Doppler imaging of orbital vessels. Rinsho Byori Feb 55(2) 170-8..

4. Baydar s, Ad.apinar B, Kebapci N etal. Australia Radio!, 2007 June 51 (3) 230-5 colour Doppler ultrasound evaluation of orbital vessels in diabetic retinopathy.

5. Arai $T$, Num.ata $K$, Tanaka $K$, etal $J$ ultrasound med. 1998 Nov; 17(11): 675 81.

6. Krasnicki P., Mariak Z, ustymowicz, proniewska-skretek E. " Assessment of blood flow in the ocular circulation in type
2 DM with colour Doppler imaging" klin oczna 2006; 108 (7-9); 294-8.

7. Mackinno.n JR, MCKillop G,0 Brienc, swa K, Nelson P. Acta ophthalmol scand, 2000 Aug;386-9 Colour Doppler imaging of the ocular circulation in diabetic retinopathy.

8. Gracner T. Ophthalmologica 2004 jul-aug; 218 94); 237-42. Ocular blood flow velocity determined by colour Doppler imaging in diabetic retinopathy.

9. JIANG shi Jun, Luo Hai — xiang, JIANG. Yan - ling. Journal of clinical ophthalmology $2006-06$. Study of ophthalmic and central retinal arteries blood flow for the diabetic patients.

10. Gil Hernandez MA, Abrerk Reyes P, Quintero M, Ayala E arch SOC ESP oftalmol. 2001 mar, 76 (3) $175-80$. Doppler ultrasound in type 1 diabetes.

11. Tamaki Y, Nagahara M, Yamashita H. Kikuchi M. Nippon Ganka Gakkai Zasshi 1993; 97 (8); 961-966. Analysis of blood flow velocity in OA by colour Doppler imaging.

12. Galina dimitrova. European ophthalmic review 2011, 5 (1); 16-9 colour Doppler imaging of ocular and orbital blood vessels in retinal disease.

13. JIANG shi Jun, Luo Hai-xiang Yan-ling ; Journal of clinical ophthalmology 200606;' Study of ophthalmic and central retinal arteries blood flow for diabetic patients.

14. Ma Jialian, Yin Yiniin, chen Wei etal. Journal of Xi'an Medical university (chineese) 1999-01. The changes of ocular and orbital haemodynamics in patients with diabetes.

15. ZHANG Gai-ying. SHIMIow, GONG Lixin etal ; Journal of medical imaing 200607; Study of haemodynamics of eye fundus blood vessel in patients with diabetes mellitus with colour Doppler flow trnatna. 
16. NIE FANG, TONG Min-hui, CHE Yan.Journal of medical imaging 2005-11. Colour Doppler ultrasonoraphic study on haemodynarnic alteration of optic artery branches in patients with diabetes mellitus.

17. Gosling RG, DunbarG, King DH etal.The quantitative analysis of occlusive orbital disease by a nonintrusive ultrasonic technique. Angiology 22;52,1971

18. Guven D, Ozdemir H, Hasanreisoglu B; Haemodynamic alterations in diabetic retinopathy. Ophthalmology 103; 1245,1996.

19. Fake GT, Buzney SM, Ogasawara Hetal. Retinal circulatory abnormalities in type 1 diabetes. Invest ophthalmol vis sci $35 ; 2968,1994$.

20. GUO Haifang, LIU shandy .China Medical Herald 2009- 11.Haemodynamics study of colour Doppler ultrasound to central artery of retina of diabetics. 